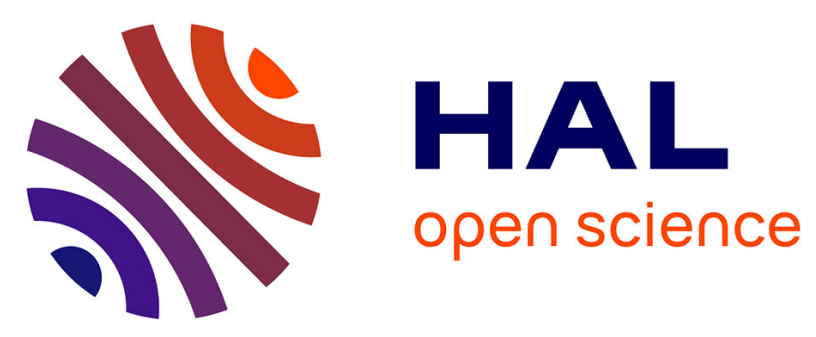

\title{
Risk analysis of structures in presence of stochastic fields of deterioration: flowchart for coupling inspection results and structural reliability
}

Franck Schoefs

\section{- To cite this version:}

Franck Schoefs. Risk analysis of structures in presence of stochastic fields of deterioration: flowchart for coupling inspection results and structural reliability. Australian Journal of Structural Engineering, 2009, 9 (1), pp.67-78. 10.1080/13287982.2009.11465011 . hal-01007262

\section{HAL Id: hal-01007262 \\ https://hal.science/hal-01007262}

Submitted on 12 Feb 2017

HAL is a multi-disciplinary open access archive for the deposit and dissemination of scientific research documents, whether they are published or not. The documents may come from teaching and research institutions in France or abroad, or from public or private research centers.
L'archive ouverte pluridisciplinaire HAL, est destinée au dépôt et à la diffusion de documents scientifiques de niveau recherche, publiés ou non, émanant des établissements d'enseignement et de recherche français ou étrangers, des laboratoires publics ou privés.

\section{(c)(1)}

Distributed under a Creative Commons Attribution| 4.0 International License 


\title{
Risk analysis of structures in presence of stochastic fields of deterioration: Flowchart for coupling inspection results and structural reliability
}

\author{
F Schoefs \\ Institute for Research in Civil and Mechanical Engineering (GeM), Nantes Atlantic University, France
}

\begin{abstract}
SUMMARY: Inspection by non-destructive testing techniques of existing structures is not perfect and it has become a common practice to model their reliability in terms of receiver operating characteristic (ROC) curves. This paper suggests a method of building ROC curves in case of random fields of defects on a structure by using polynomial chaos decomposition. Knowledge of spatial distribution of defects allows a reliability analysis to be performed. When selecting stochastic finite element analysis to solve this problem, the format is the same as the one chosen for modelling inspections results. The paper shows how to link these quantities (ie. reliability and inspection results) in a risk analysis by using polynomial chaos decomposition as a common language.
\end{abstract}

\section{INTRODUCTION}

Reassessment of existing structures generates a need for up-dated materials properties. Commonly, onsite inspections are needed and in some cases visual inspections are not sufficient for an accurate sizing or detection. For example, non-destructive testing (NDT) tools are required for the inspection of coastal and marine structures where marine growth acts as a mask. In these fields, the cost of inspection can be prohibitive and an accurate description of the onsite performance of NDT tools must be provided. Inspection of existing structures by a NDT tool is not perfect and it has become a common practice to model its reliability in terms of probability of detection (PoD), probability of false alarms (PFA) and receiver operating characteristic (ROC) curves. These quantities are generally the main inputs needed by owners of structures in view to achieve Inspection, maintenance and repair plans (IMR) (Sheils et al, 2008). The assessment of PoD and PFA comes either from inter-calibration of NDT tools or from the modelling of the probability density functions of the noise caused by the NDT imperfection and the signal. In this last case, if the noise and the signal depend on the location on the structure then PoD and PFA are spatially dependent. The decomposition on polynomial chaos $(\mathrm{PC})$ is then used to represent the underlying stochastic fields. It is then natural to perform reliability analysis in the presence of a stochastic field of defects by using the same format. It is shown that PC decomposition offers in this case a common language for the two inputs of the RBI: the modelling of inspection results and the computing of the structural reliability.

This paper presents firstly how to define PoD and PFA when damage and detection are stochastic fields, ie. spatially dependent (section 2). Section 3 deals with PC decomposition of the marginal distributions of the stochastic fields obtained at given locations along the structure. It is shown how to identify the coefficients of the PC decomposition from data.

However, it has been shown (Rouhan \& Schoefs, 2003) that PoD and PFA cannot be directly linked with consequences when an IMR policy has been defined. From the decision theory and a Bayesian description of inspection results, it is shown in section 4 how to introduce new events for risk-based inspection (RBI). Expressions of their likelihood as functions of PoD, PFA and $\gamma$, the probability of defect presence are presented. Results are then generalised to use ROC curves for RBI. By knowing the spatial stochastic field for the signal and the noise, ROC curves are deduced in each point of the field. This section is illustrated with the ultrasonic measurement of the spatially distributed corrosion of a sheet pile in a marine environment. 
Finally, section 5 gives the last output needed for risk assessment based on reliability analysis: the computing of the probability of failure. From loading modelling and by knowing the stochastic field of defects, a stochastic computation can be performed in view to estimate the spatial distribution of probability of failure. This section ends with a complete application of risk analysis of a corroded sheet-pile in steel.

\section{ASSESSMENT OF STOCHASTIC FIELDS OF DEFECTS THROUGH NDT TOOLS}

\subsection{Hazards and uncertainties when assessing defect sizes on structures}

Let us consider the assessment of a defect $d$ through a NDT tool. Inspection is generally not perfect. We consider the case where harsh conditions affect the measurement of the $d$. Then, depending on the environment and the location of the inspected area, the measurement of the size is affected by hazards. We model this quantity as a stochastic field $d(X, t, \theta)$, where $X$ denotes the spatial coordinates, $t$ the time and $\theta$ the hazard. When assessing this defect, the chain of measurement (accessibility of the inspected area, calibration of NDT tool, experience and tiredness of the operator) introduces uncertainties, called noise in the signal processing theory (Rouhan \& Schoefs, 2003). The result of the inspection, ie. the measured defect size, is a stochastic field denoted $\hat{d}(X, t, \theta)$. In section $2.2, d(X, t, \theta)$ and $\hat{d}(X, t, \theta)$ are written as $d$ and $\hat{d}$ for simplicity.

\subsection{Modelling capability of NDT tools in a probabilistic context}

The most common concept that characterises inspection tool performance is the PoD. Let $a_{d}$ be the minimal defect size, under which it is assumed that no detection is done. Parameter $a_{d}$ is called the detection threshold in the following. Thus, the PoD is defined as:

$\mathrm{PoD}=P\left(\hat{d} \geq a_{d}\right)$

The detection threshold $a_{d}$ is a deterministic parameter or a random variable. This definition implies that PoD is a monotonic increasing function.

Detection theory allows for the introduction of another useful quantity, the PFA. From the signal theory, $\hat{d}$ is called the complete signal or "signal + noise" with a probability density function (pdf) $f_{S N^{*}}$ The real defect size $d$ is unknown and it is deduced from the knowledge of the noise $\eta$ by the relationship $\hat{d}=d+\eta$. The pdf of noise is denoted $f_{N}$. Noise depends on environmental conditions, human interference and the nature of what is being measured. Let's assume that noise and signal amplitudes are independent random variables, then
PoD and PFA have the following expressions (2) and (3):

$$
\begin{aligned}
& \mathrm{PoD}=\int_{a_{d}}^{+\infty} f_{S N}(\hat{d}) \partial \hat{d} \\
& \mathrm{PFA}=\int_{a_{d}}^{+\infty} f_{N}(\eta) \partial \eta
\end{aligned}
$$

where $\delta$ denotes the increment. Figure 1 illustrates the corresponding pdf in the case where variables are normally distributed and the computation of PFA and PoD for a given detection threshold.

For a given detection threshold, the couple (PoD, PFA) allows the performance of a NDT to be defined; this is the ROC. This couple can be considered as the coordinates of a point. Let us consider that $a_{d}$ is unknown and takes values in the range $[-\infty ;+\infty]$, this point belongs to a curve called ROC curve. It is a parametric curve defined by equations (2) and (3) with parameter $a_{d}$.

The ROC curve, which is plotted in figure 2, is computed using the pdf presented on figure 1 . From a theoretical point of view, this is a convex curve corresponding to a monotonically increasing function, always lying above the diagonal line, where the first derivative is closely linked to the sensitivity of the receiver (Arques, 1982; Fücsök et al, 2000). The diagonal line running from the lower left to the upper right (curve "PoD=PFA") is the line of no "performance", since in that case the inspection PoD equals PFA whatever the detection threshold (Rouhan \& Schoefs, 2003).

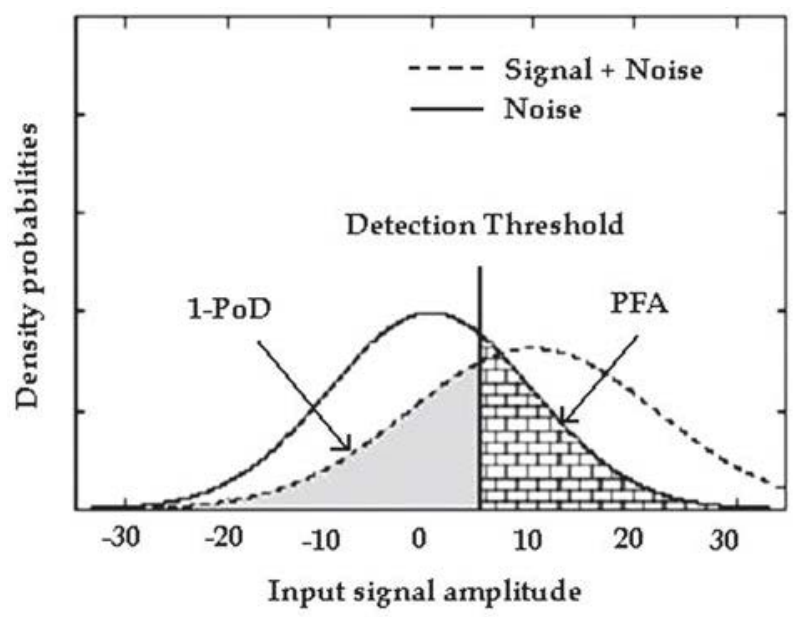

堅 Probability of false alarm

Probability of a miss detection

Figure 1: Illustration of PoD and PFA (signal and noise normally distributed) (Rouhan \& Schoefs, 2003). 


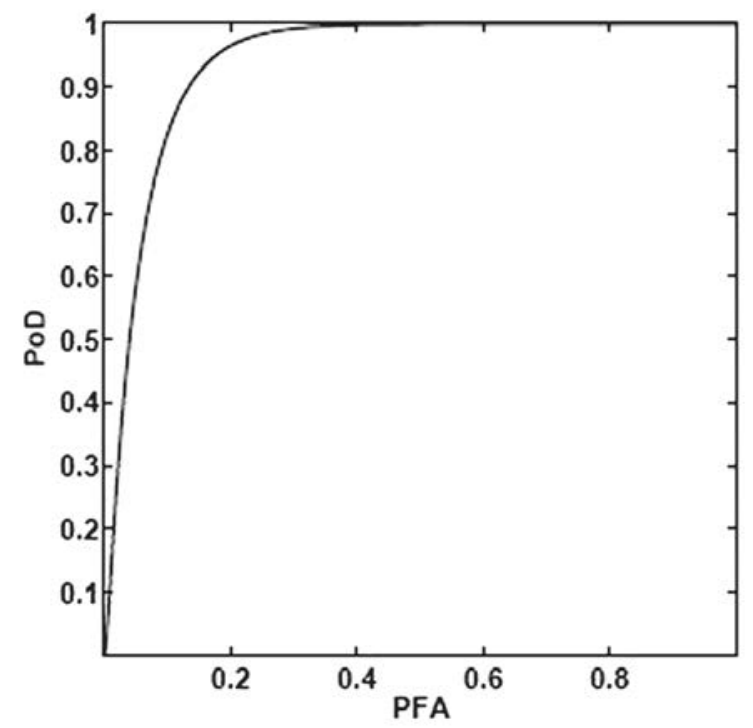

Figure 2: $\quad$ Receiver operating characteristic curve.

Looking for the best detection performance, the PoD should always take larger values than the PFA (low noise sensitivity). We have then PoD $\geq$ PFA. When analysing ROC curves, one must keep in mind that the PFA depends on the noise and detection threshold only. It does not depend on defect unless the noise depends on defect size. That is the case for instance if the operator adjusts the device to detect smaller defects when the current adjustment does not give any signal. The PoD is a function of the detection threshold, the defect size and the noise. Thus, for a given detection threshold, the PFA is a constant, but the PoD is an increasing function of the defect size. The ROC curve is a fundamental characteristic of the NDT tool performance for a given defect size. A perfect tool is represented by a ROC curve reduced to a single point whose coordinates are $(\mathrm{PoD}, \mathrm{PFA})=(1,0)$. The distance between this "best performance point" and the ROC curve is a measure of the NDT ability (Schoefs \& Clement, 2004); we call it NDT-PI (NDT Performance Index). Theoretical ROC curves are presented in Rouhan \& Schoefs (2003), where each one is obtained for several signal/ noise ratios of a NDT tool.

When assessing a ROC curve, two approaches can be considered:

- the statistical approach, which aims to provide discrete values for PFA and PoD and requires the knowledge of the real size; this is performed within specific inter-calibration projects such as the ICON project in the field of offshore platform analysis (Barnouin et al, 1993), when on-site measurements on real structures are costly or when on-site conditions are affected by a lot of factors as for the detection of corrosion pitting (Pakrashi et al, 2008)

- the probabilistic approach, which requires a modelling of the (signal + noise) and noise pdf; it is generally preferred when the cost of on-site inspections is reasonable and allows a rich data base of information to be accessed.

We follow the second approach in this paper. Note that the PFA is named PFI (probability of false indication) too. The definition of discrete values for PFI is generally expressed as a percentage of false indications on the inspected length (Silk, 1996; Rudlin et al, 2006). Finally, as the number of samples is limited, authors provide confidence bounds: $90 \%$ PoD for example (Barnouin et al, 1993; Rudlin et al, 2006).

In some cases, the performance of NDT tools depends on the location of the point to be inspected on the structure. For example, when locations of defects are on welded joints in the sea, the corresponding PoD and PFA should be changed according to the access, the luminosity and the wave shaking for instance. When defects are continuous fields on the structure, the PoD and PFA should be indexed by the coordinates $X$ of the inspected point $M$. We consider here that the defect is induced by a deterioration mechanism indexed by time $t$ and can be modelled by a stochastic field: $d(X, t, \theta)$.

\subsection{Definitions of PoD and PFA for stochastic deterioration model}

As described in the previous section, after inspection with a NDT tool, the size of the defect $d(X, t, \theta)$ becomes $\hat{d}(X, t, \theta)$. Then the noise $\eta(X, t, \theta)$ is defined from the knowledge of these two stochastic fields by equation (4).

$\eta(X, t, \theta)=\hat{d}(X, t, \theta)-d(X, t, \theta)$

For each location $X$ and at each time $t$, the PoD and PFA are, respectively, computed from equations (2) and (3). Thus the PoD and PFA, as well as the ROC curves, are functions indexed by $X$ and $t$. We denote $\operatorname{PoD}(X, t)$ and $\operatorname{PFA}(X, t)$ as functions of time and space. The field $\hat{d}$ at a given time $t$ being assessed from inspection, the computation of $\operatorname{PoD}(X, t)$ and $\operatorname{PFA}(X, t)$ requires the knowledge of one of the other stochastic fields in equation (4): $d(X, t, \theta)$ or $\eta(X, t, \theta)$. Two situations can be considered:

(i) The noise is known because it is constant whatever the location of the NDT tool on the structure or because it is constant on given areas on the structure. It is generally time invariant and zero mean.

(ii) The real size is known because it has been measured before on-site inspections as in the ICON project (Barnouin et al, 1993) or because an assumption is made.

In both situations, the definition of continuous spatial functions needs the complete characterisation of the stochastic fields by their joint distribution. Practically, no models of joint distribution are available for onsite inspection and almost all NDT tools give data on 
specific locations; thus, only marginal distributions can be assessed. Moreover, the owner generally does not base its inspection planning on RBI methods; then the inspection campaign aims to give a global overview of the state of the structure. Thus the distance between measurements is generally larger than the distance of correlation and additional assumptions on the structure of correlation for the stochastic fields are needed.

Finally, note that the knowledge of ageing laws for $d$ allows predicting evolution of ROC curves with time. This can be implemented in a RBI method for optimising the period between inspection, as well as the location of inspections. For example, for corrosion processes, several models are available (Melchers, 2003; Paik et al, 2003a; Paik et al, 2003b; Guedes Soares \& Garbatov, 1999). In the field of uniform corrosion, spatially dependent ROC curves are available (Schoefs et al, 2007a).

\section{3 \\ MODELLING RANDOM FIELDS OF DEFECTS FROM NDT MEASUREMENTS}

Only the spatial dependence is addressed here. We suggest a representation with PC decomposition for modelling marginal distributions or random fields. It allows for the systematic identification of random variables or random fields. We choose the estimate of maximum likelihood for the identification of PC decomposition (Desceliers et al, 2007). This method has already been applied for the identification of random variables from structural monitoring (Schoefs et al, 2007b). The question is to identify the coefficients $d_{i}, \hat{d}_{i}$ and $\eta_{i}$ of the one-dimensional PC decomposition for every random variable. For generality, let us denote $x(\theta)$ as the random variable and $x_{i}$ as the PC coefficients. Equation (5) gives the general form of a one-dimensional PC decomposition.

$x(\theta)=x(\xi(\theta))=\sum_{i=0}^{p} x_{i} h_{i}(\xi(\theta))$

where $p$ is the order of the PC decomposition; $\xi(\theta)$ is the Gaussian germ, ie. a standardised normal variable; and $h_{i}$ is the Hermite polynomial of degree $i$. By using the maximum likelihood method, coefficients $x_{i}$ are solutions of the optimisation problem:

$L(\mathbf{x})=\underset{\mathbf{x}}{\arg \max } L(\mathbf{x})$

where $\mathbf{X}$ is the vector of components $x_{i}\left(\mathbf{x}=\left[x_{0^{\prime}}, \ldots, x_{p}\right]\right)$ with dimension $(p+1)$, and $L$ is the likelihood function:

$L(\mathbf{x})=\prod_{j=1}^{N} p_{x}\left(x\left(\theta_{j}\right) ; \mathbf{x}\right)$

where $x\left(\theta_{i}\right)$ is the $j^{\text {th }}$ realisation (here measurement) of $x$; and $p_{x}(. ; \mathbf{x})$ is the probability distribution of $x$, parameterised by $\mathbf{x}$.
The likelihood function (7) takes very fair values close to the numerical precision. Then the problem (6) is replaced by equation (8):

$-\log (L(\mathbf{x}))=\underset{\mathbf{x}}{\arg \min }(-\log (L(\mathbf{x})))$

The algorithm for solving the optimisation problem (8) is detailed in Schoefs et al (submitted) and only the main steps are described here. Basically, the question is to build a solution by stating conditions (9):

$\left\{\begin{aligned} x_{0} & =\mu_{0} \\ \operatorname{Var}(x(\xi(\theta)) & =\sum_{i=1}^{p} x_{i}^{2}=\sigma_{x}\end{aligned}\right.$

where $\mu_{x}$ and $\sigma_{x}$ are, respectively, the statistical average and standard deviation of variable $x(\theta)$. They are computed from the $N$ measurements $x\left(\theta_{j}\right)$. In equation (9), the first condition reduces the number of unknown PC coefficients to $p$ and the second one facilitates the search for other coefficients on an hyper-sphere with radius $\sigma_{x}$. Moreover, by denoting $x_{i}^{*}$ the quantity $x_{i} / \sigma_{x^{\prime}}$ the second condition of (9) becomes equation (10):

$\sum_{i=1}^{p}\left(x_{i}^{*}\right)^{2}=1$

This new condition facilitates the search for $p$ coefficients $x_{1}^{*}, \ldots x_{i}^{*}, \ldots x_{p}^{*}$ on a hyper-sphere with radius 1 . This last condition is interesting for the optimisation process. Let us now consider a PC of order 3. To parametrise the hyper-sphere, we can introduce two angular parameters $\phi_{1}$ and $\phi_{2}$ :

$$
\begin{aligned}
& \left\{\begin{array}{l}
x_{1}^{*}=\cos \left(\varphi_{1}\right) \cos \left(\varphi_{2}\right) \\
x_{2}^{*}=\cos \left(\varphi_{1}\right) \sin \left(\varphi_{2}\right) \\
x_{3}^{*}=\sin \left(\varphi_{1}\right)
\end{array}\right. \\
& \text { with } \varphi_{1} \in\left[-\frac{\pi}{2} ; \frac{\pi}{2}\right] \text { and } \varphi_{2} \in[-\pi ; \pi]
\end{aligned}
$$

A two-step optimisation flowchart is used solving equation (8) by knowing equation (11):

- a first localisation of the minimum is found through Monte Carlo simulations (size 100 and 1000, respectively, for PC of order 2 and 3)

- starting from this point, the Nelder-Mead Simplex Method is used (Lagarias et al, 1998).

This process allows us to avoid a pseudo-convergence around minimum values. Figure 3(a) presents the fitting of distributions in the case of uniform corrosion of piles in harbours for the two variables: measured loss of thickness $\hat{d}$ and noise $\eta$ with PC for several orders $p$. Figure 3(b) plots iso-values of $-\log (L)$ for PC chaos of order 3 , in the space $\left(\phi_{1}, \phi_{2}\right)$. ROC curves are then deduced from integration of these decompositions. Thus both PoD and PFA are functions of polynomial coefficients $x_{i}$. Denoting $\hat{\mathrm{d}}$ and $\eta$ the vectors, respectively, of $i^{\text {th }}$ components $\hat{d}_{i}$ and $\eta_{i^{\prime}}$ expressions of PoD and PFA as functions of PC 


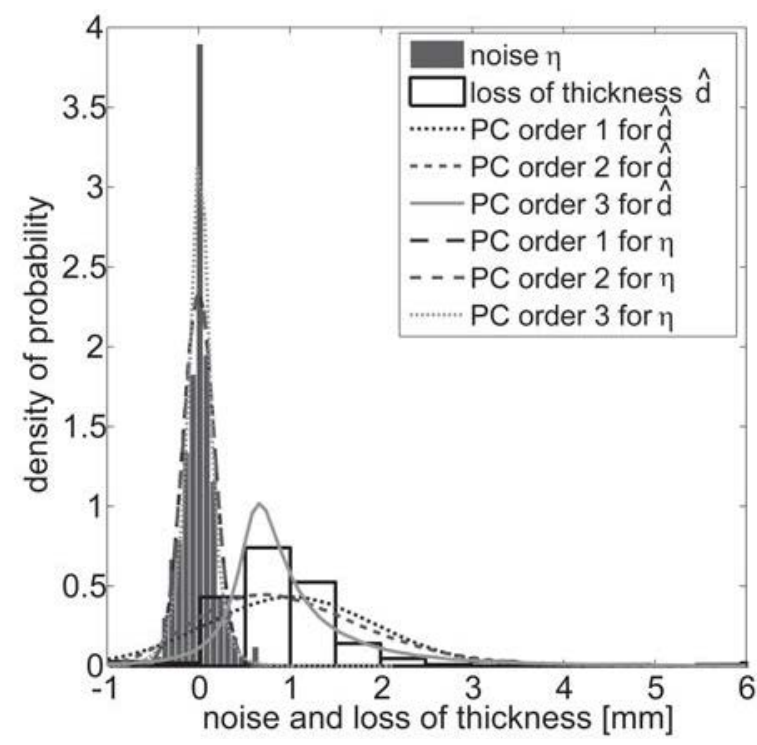

Figure 3(a): Fitting of loss of thickness and noise with polynomial chaos for several orders $p$ (corrosion of piles in harbours).

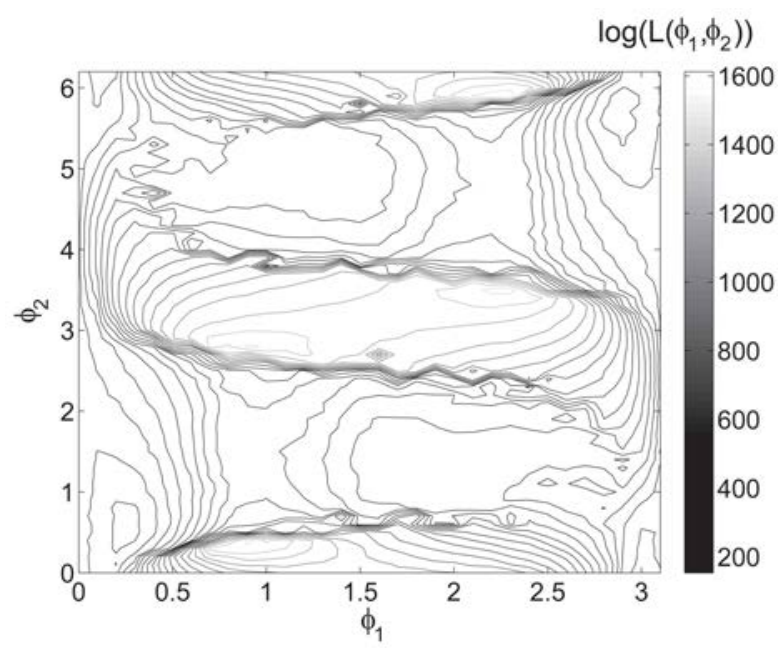

Figure 3(b): Representation of $-\log (L)$ in the space $\left(\phi_{1}, \phi_{2}\right)$ for PC chaos of order 3 .

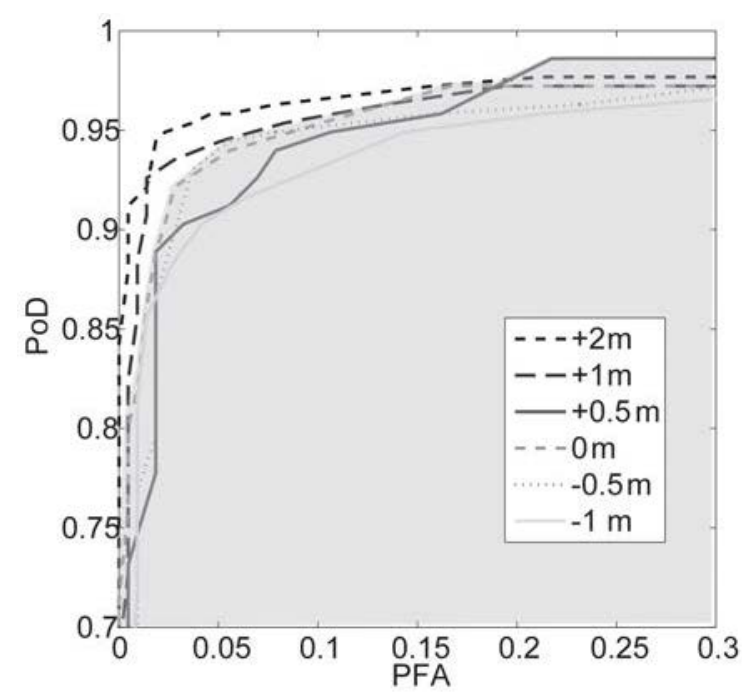

coefficients are presented in equations (12) and (13). When $\hat{d}$ and $\eta$ are independent, a germ of dimension 2 is needed respectively $\left(\xi=\left(\xi_{1}, \xi_{2}\right)\right.$. Note that the PC decomposition of signal $d$ is obtained from equation (4) by knowing $\hat{d}, \eta, \xi_{1}$ and $\xi_{2}$.

$\mathrm{PoD}=\sum_{i=0}^{p}\left(\hat{d}_{i} \Im_{i}\left(a_{d}\right)\right)$

$\mathrm{PFA}=\sum_{i=0}^{p}\left(\eta_{i} \mathfrak{\Im}_{i}\left(a_{d}\right)\right)$

with $\mathfrak{J}_{i}\left(a_{d}\right)=\int_{-\infty}^{a_{d}} h_{i}(u) p_{\xi} d u$

where $p_{\xi}$ is the probability density function of $\xi$ (standard normal pdf). Practically, integrals $\mathfrak{I}_{i}\left(a_{d}\right)$ in equations (12) and (13) are computed through Monte-Carlo simulations using $10^{6}$ samples. These quantities are independent of the study: they can be preprocessed once for all and used for each application.

The stochastic field of corrosion for sheet piles in harbours is spatially indexed by the vertical abscissa only (Schoefs et al, 2007a). ROC curves at six depths between $-1 \mathrm{~m}$ and $+2 \mathrm{~m}$ along a pile are plotted in figure 4 for a PC chaos of order 3 . Abscissa $(+1 \mathrm{~m})$ and $(+2 \mathrm{~m})$ represent points located in the tidal area where others refer to locations in the immersion area.

Note that the identification of a variance-covariance matrix is generally more complex due to the distance of inspected points that is much larger than the correlation length as mentioned previously in section 2.3. In this case we suggest using independent germs $\xi_{i}(\theta)$ for each inspected point and to interpolate PC coefficients between two inspected points (14).

$d_{i}(X, \theta)=\sum_{j} \phi_{k}(X) \cdot d_{i}\left(X_{j}\right)$

where $\phi_{k}(X)$ are linear functions for interpolation from knowledge of coefficients at coordinate

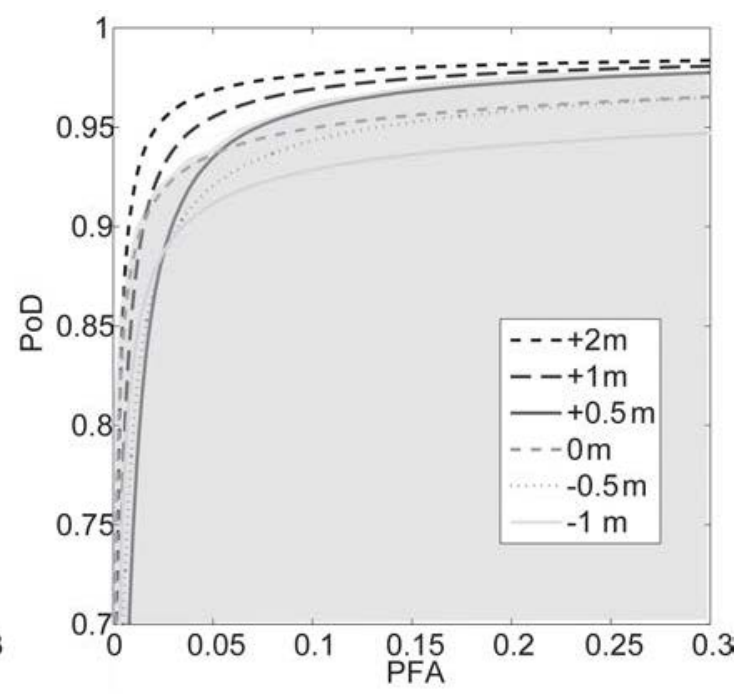

Figure 4: Comparison between ROC curves coming from polynomial chaos with order 3 identification (left) and experimental data (right) for several inspections in depth. 
$X_{i}$. Note that this representation needs at least measurements where the average trajectory of the process takes its minimum and maximum value. In the case of corrosion of coastal structures, shape of profiles and position of extreme values are generally known and inspections are carried out at these positions (see figure 5). In view to compare this method with another one based on the fitting with a classical pdf, we used the NDT-PI: it is the distance between the best performance point in (PFA, PoD) space of coordinates $(0,1)$ and the ROC curve. Table 1 gives a comparison between the NDT-PI obtained with predefined pdf (table 1(a)) (Normal, Generalised Extreme Value (GEV), Student pdf) and PC identifications (table $1(\mathrm{~b})$ ) at a given position along the vertical axis $(X=+2 \mathrm{~m})$. The value of NDTPI deduced from the original curve (experimental) being 0.054 , only PC chaos of order 3 allows us to reach this value $(0.052)$ when both lower order PC decomposition and predefined pdf leads to values of around 0.075 . The tables give the detection threshold $a_{d}$ at the best performance point. Values of coefficients are given at each inspected level on figure 6 for PC decomposition of order 3.

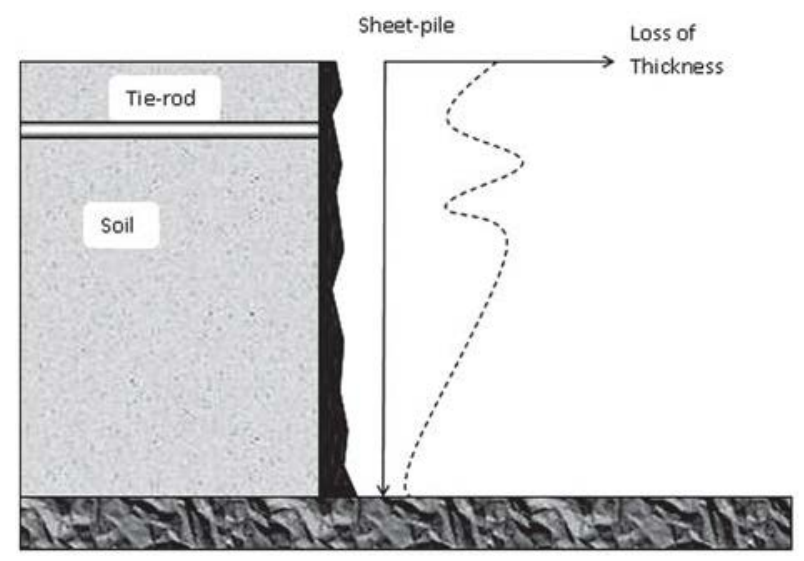

Figure 5: Corroded sheet-pile and profile of the loss of thickness.
We notice that the normal pdf and order 1 PC decomposition are the same, leading to the same result.

\section{RISK-BASED DECISION AID-TOOLS BASED ON INSPECTION RESULTS}

Rouhan \& Schoefs (2003) further developed this methodology by focusing on the probability that a defect exists after an inspection has been carried out. The PoD is the probability that an existing defect is detected, whereas the decision is based on the event that a defect exists, given the results of an inspection. We consider the events:

Table 1(a): Values of NDT-PI and corresponding detection threshold for predefined pdf.

\begin{tabular}{|l|c|c|}
\hline \multirow{2}{*}{ ROC curves } & \multicolumn{2}{|c|}{$X=\mathbf{+ 2} \mathbf{~ m}$} \\
\cline { 2 - 3 } & NDT-PI & $\boldsymbol{a}_{\boldsymbol{d}}$ \\
\hline Experimental & 0.054 & 0.25 \\
\hline Normal & 0.079 & 0.26 \\
\hline GEV & 0.081 & 0.29 \\
\hline Student & 0.072 & 0.25 \\
\hline
\end{tabular}

Table 1(b): Values of NDT-PI and corresponding detection threshold for PC decomposition (order 1 to 3 ).

\begin{tabular}{|l|c|c|}
\hline \multirow{2}{*}{ ROC curves } & \multicolumn{2}{|c|}{$X=+\mathbf{2 ~ m}$} \\
\cline { 2 - 3 } & NDT-PI & $\boldsymbol{a}_{\boldsymbol{d}}$ \\
\hline Experimental & 0.054 & 0.25 \\
\hline PC order 1 & 0.079 & 0.26 \\
\hline PC order 2 & 0.072 & 0.026 \\
\hline PC order 3 & 0.052 & 0.026 \\
\hline
\end{tabular}

Figure 6: $\quad$ PC coefficients (order 3) identified at several depths. 
- the real state $\delta$ : " $\delta=1$ " means that "defect is present"

- " $D(\delta)$ " the decision on this state after inspection: " $D(\delta)=1$ " means that "defect is detected".

From Bayesian point of view, PoD can then be defined as the conditional probability of the event " $(D(\delta)=1 \mid \delta=1)$ ". The conditional probability of the event of interest " $(\delta=1 \mid D(\delta)=1)$ " can be evaluated using Bayes Theorem. It is subsequently introduced into cost functions, which are used to investigate the effect of cost overrun due to inaccurate inspection results. Four events $E_{i}(i=1: 4)$ are then defined. For example, the expression of the probability of event $E_{3} "(\delta=1 \mid D(\delta)=0)$ " is given in equation (15). This event is of first importance in risk analysis because it can lead to a failure.

$$
P\left(E_{3}\right)=\frac{(1-\mathrm{PoD}) \gamma}{(1-\mathrm{PoD}) \gamma+(1-\mathrm{PFA})(1-\gamma)}
$$

Other events are:

- $E_{1}$, is the event "absence of defect knowing no detection" or " $\delta=0 \mid D(\delta)=0)$ "

- $E_{2^{\prime}}$ is the event "absence of defect knowing a detection" or " $\delta=0 \mid D(\delta)=1)$ "

- $E_{4^{\prime}}$ is the event "presence of defect knowing a detection" or " $(\delta=1 \mid D(\delta)=1)$ ".

They can be associated to costs and subsequently introduced in a complete RBI planning (Sheils et al, 2008). The occurrence of these events also depends on another parameter, $\gamma$, the probability of presence of a defect. The probability density function of $\gamma$ is related to the natural size of existing defects and their spatial variation (which comes from expert judgment or an historical data base).

An alternative approach is to introduce a parameter known as the probability of indication (PoI):

$\operatorname{PoI}(d)=\operatorname{PoD}(d)+(1-\operatorname{PoD}(d)) \operatorname{PFI}$

where PFI is the probability of false indication (Straub $\&$ Faber, 2003). The parameter PFI must be defined relative to a specific size of the inspected area (Straub \& Faber, 2003).

From equations (12) and (13), $P\left(E_{i}\right)$ and PoI are expressed as functions of spatial dependant quantities $\hat{d}$ and $\eta$. Figure 7 gives the evolution of $P\left(E_{2}\right)$ and $P\left(E_{3}\right)$ for three assumptions of expert judgement and the result after selection of a standard expert judgement. This factor strongly affects the shape of these curves.

\section{RELIABILITY ANALYSIS BASED ON STOCHASTIC FINITE ELEMENT IN CASE OF RANDOM FIELDS OF DEFECTS}

The stochastic (or probabilistic) finite element method refers to finite elements methods that account for uncertainties in the geometry or material properties of a structure, as well as the applied loads. Since the 1980s, quite a lot of developments have been made. They are mainly concerned with uncertainties of materials properties or hazards in loading. The question of the random geometry due to defects during building or the effect of ageing laws (corrosion) is still a challenge. Methods such as X-SFEM are efficient in this context (Nouy et al, 2007; 2008a; 2008b; Clément et al, 2008). In this paper, the computation is based on the so-called non-intrusive stochastic finite element analysis (Berveiller, 2005). We consider in this section that the loss of thickness $d(X, t, \theta)$ can be introduced in reliability through "macro" variables, ie. quantities such as diameter, section or inertia are explicit function of $d$. Then no specific method that deals with random geometries such as X-SFEM is needed.

Firstly, a quantity of interest or a limit state is selected. Here, we are interested in displacement of each point of the structure displacements $u(X)$. The limit state is then expressed: $G(X)=u(X)-u_{c}$, where $u_{c}$ is the acceptable critical displacement. The structural computation is made by using deterministic software. The question is then to find an approximation $\tilde{u}(X, \xi)$ of the PC decomposition $u(X, \xi)$. By knowing the decomposition of basic input variables such as $d(X, t, \theta)$, on a PC decomposition with germ $\xi, \tilde{u}(X, \xi)$ is expressed in equation (17) and can be assessed by computing $u_{\alpha}$ through the quadrature formula (18).

$$
\begin{aligned}
& \tilde{u}(\theta)=u(\xi(\theta))=\sum_{\alpha} u_{\alpha} H_{\alpha}(\xi(\theta)) \\
& u_{\alpha}=\int_{\theta} H_{\alpha}(\theta) u(\theta) d P(\theta)=E\left(H_{\alpha} u\right) \approx \sum_{k} \omega_{\alpha} H_{\alpha}\left(\theta_{k}\right) u\left(\theta_{k}\right)
\end{aligned}
$$

where $\alpha$ is a multi-index, $P$ is a measure of probability associated with $\xi$, and $H_{\alpha}$ is an Hermite polynomial associated to this multi-index. $\theta_{k}$ are the elementary events where the deterministic computation is needed (generally called point of integration or Gauss points in the case of normal measure of probability) and $\omega_{k}$ is the corresponding weights associated with the measure of probability $P$.

A post-processing allows the computation of the probability of failure $P_{f}$ to be carried out with very low computational costs. This is of primary interest when carrying out risk analysis where a good assessment of $P_{f}$ is needed.

For illustration, we can consider an application where an explicit form of the solution can be obtained by the static balance of the structure assuming an elastic behaviour of the material (see figure 5).

Considering here a limit state relying on yield momentum, the probability of failure with depth is plotted on figure 8 , with the loss of matter on figure 6 and the configuration of loading on figure 5 . 

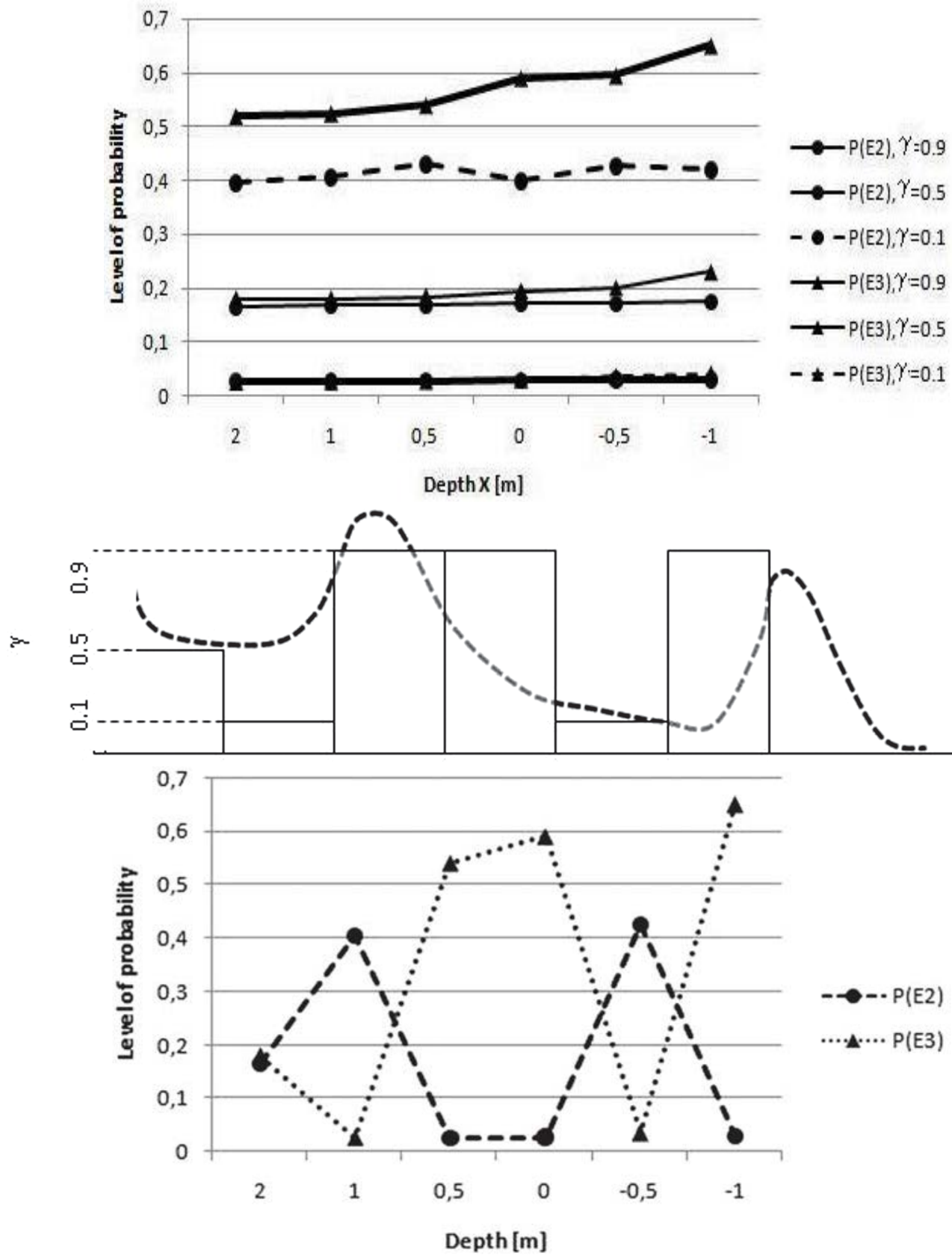

Figure 7: Evolution of $P\left(E_{2}\right)$ and $P\left(E_{3}\right)$ with depth (top) for several expert judgements $\gamma$ (in bold line 0.9, in fine line 0.5 and in dotted line 0.1 ); standard expert judgement (middle); and corresponding evolution of $P\left(E_{2}\right)$ and $P\left(E_{3}\right)$ with depth with this standard judgement (bottom).

\section{6}

\section{CONCLUSION: FLOWCHART FOR COUPLING RESULTS OF INSPECTION AND STRUCTURAL RELIABILITY}

Sections 4 and 5 provide the main steps for a flowchart that couples the results of inspection and structural reliability with a formulation based on PC decomposition. From inspection results and noise modelling, it is based on the PC decomposition of basic variables describing the risk based modelling of inspection results $\hat{d}(X, t, \theta)$ and $\eta(X, t, \theta)$ on the one hand, and a PC decomposition of geometrical basic variables for the reliability study $d(X, t, \theta)$ on the other hand. The corresponding terms of risk analysis $P\left(E_{3}\right)$ and $P_{f}$ can be assessed. The flowchart in figure 9 resumes the steps of methodology. When considering only event E3 (there is a defect knowing we do not detect it), which leads to failure and the probability of failure, figure 8 presents the result of the integrated risk analysis: the evolution of risk with depth. The PC decomposition is shown to be an interesting common language between reliability analysis and RBI when stochastic fields of degradation occur. Note that quite a lot of uncertainties and hazards are introduced:

(i) The natural hazard of the corrosion process: $d(X, t, \theta)$

(ii) The uncertainty in the inspection results from on-site measurements: $\eta(X, t, \theta)$

(iii) The uncertainty of the previous knowledge on the corrosion process: $\gamma(X, t)$. 

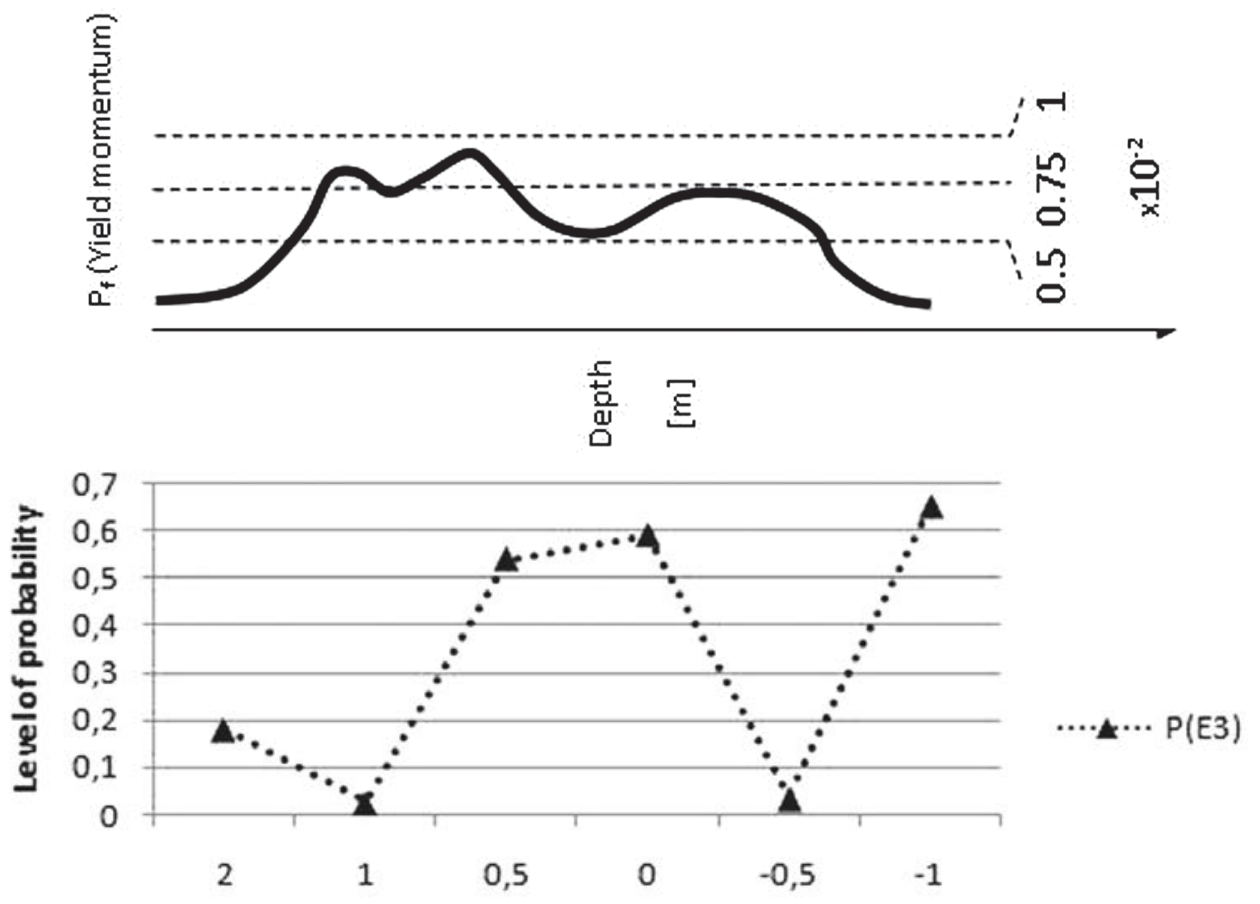

Depth [m]

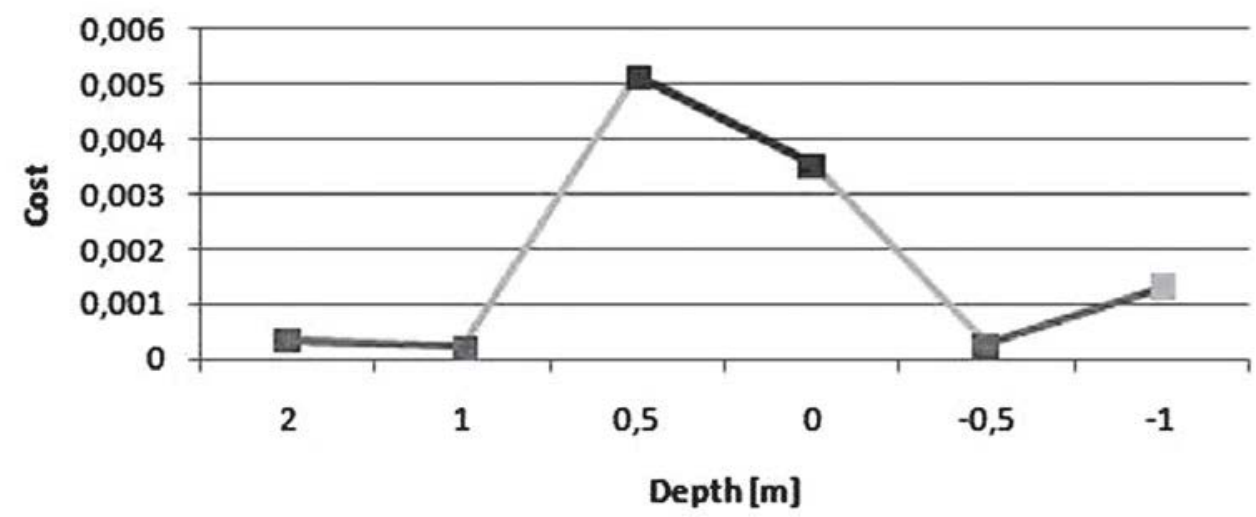

Figure 8: Evolution of probability of failure and selected $P\left(E_{3}\right)$ with depth (top and middle), and result in terms of risk (bottom).

\section{ACKNOWLEDGEMENTS}

The author is grateful for the support received from European Community within the Interreg IIIB project MEDACHS (2005-2007) and for the contribution of Alexandre Clement (Nantes Atlantic University).

\section{REFERENCES}

Arques, P. Y. 1982, Decisions en traitement du signal, Editions Masson, Paris.

Barnouin, B., Lemoine, L., Dover, W. D., Rudlin, J., Fabbri, S., Rebourcet, G., Topp, D., Kare, R. \& Sangouard, D. 1993, "Underwater inspection reliability trials for offshore structures", in Proceeding of the $12^{\text {th }}$ International Conference on Offshore Mechanics and Arctic Engineering, Vol. 3B, pp. 883-890.

Berveiller, M. 2005, "Stochastic finite element methods: intrusive and non-intrusive approaches for reliability analysis", $\mathrm{PhD}$ thesis, l'Université Blaise Pascal (Clermont-Ferrand), October.

Clément, A., Schoefs, F. \& Nouy, A. 2008, “Reliability of corroded historical lift-cranes in coastal area", Proc. of $1^{\text {st }}$ International Conference on Applications Heritage and Constructions in Coastal and Marine Environment, (MEDACHS'08), 28-30 January, Lisbon, Portugal.

Desceliers, C., Soize, C. \& Ghanem, R. 2007, "Identification of chaos representations of elastic properties of random media using experimental vibration tests", Computational Mechanics, May, Vol. 39, No. 6, pp. 831-838.

Fücsök, F., Muller, C. \& Scharmach, M. 2000, “Human factors: the NDE reliability of routine radiographic film evaluation", in Proceedings of the $15^{\text {th }}$ World Conference on Non Destructive Testing, Vol. 3.

Guedes Soares, C. \& Garbatov, Y. 1999, “Reliability of maintained corrosion protected plates subjected to 


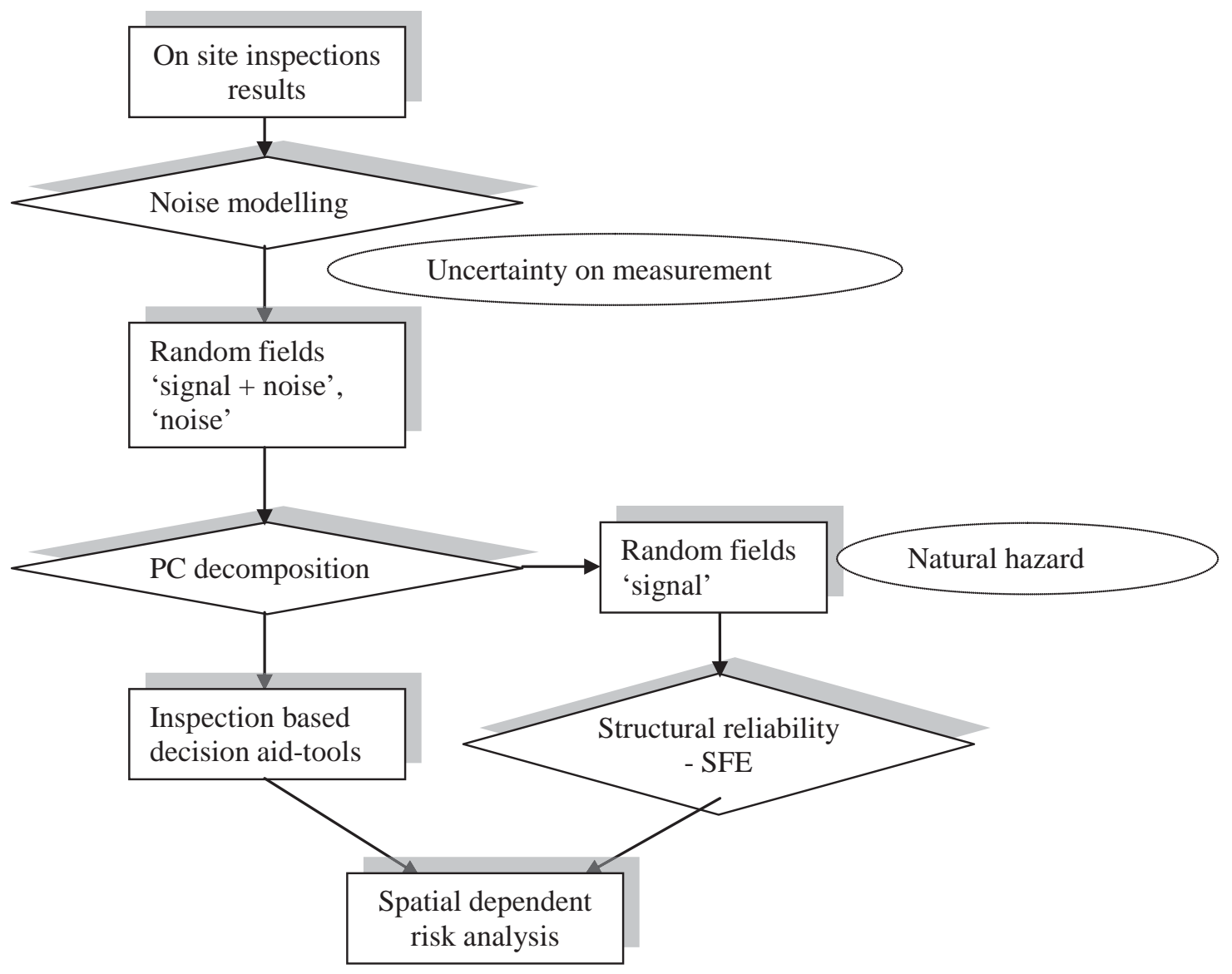

Figure 9: Flowchart for coupling results of inspection and structural reliability.

non-linear corrosion and compressive loads", Marine Structures, Vol. 12, pp. 425-445.

Lagarias, J. C., Reeds, J. A., Wright, M. H. \& Wright, P. E. 1998, "Convergence Properties of the Nelder-Mead Simplex Method in Low Dimensions", SIAM Journal of Optimization, Vol. 9, pp. 112-147.

Melchers, R. E. 2003, "A new model for marine immersion corrosion in structural reliability assessment", International Conference on Applications of Statistics and Probability in Civil Engineering (ICASP'03).

Nouy, A., Schoefs, F. \& Moës, N. 2007, “X-SFEM: a computational technique based on X-FEM to deal with random geometry", Revue Européenne de Mécanique Numérique/European Journal of Computational Mechanics, special issue on "New trends in the development of the X-FEM", Vol. 16, No. 2, pp. 277-293.

Nouy, A., Clément, A. \& Schoefs, F. 2008a, "An eXtended Stochactic Finite Element Method to deal with geometrical uncertainties in structural analysis", $8^{\text {th }}$ World Congress on Computational Mechanics (WCCM VIII) and $5^{\text {th }}$ European Congress on Computational Methods in Applied Sciences and Engineering (ECCOMAS 2008), Venice, Italy, 30 June to 5 July.
Nouy, A., Clément, A., Schoefs, F. \& Moës, N. 2008b, "eXtended Stochastic Finite Element methods for solving partial differential equations on random domains", Computer Methods in Applied Mechanics and Engineering (CMAME), to be published.

Paik, J. K., Lee, J. M., Park, Y., Hwang, J. S. \& Kim, C. W. 2003a, "Time-variant ultimate longitudinal strength of corroded bulk carriers", Marine Structures, Vol. 16, pp. 567-600.

Paik, J. K., Vincent, A. K. \& Thayamballi, A. K. 2003b, Ultimate Limit State Design of Steel-Plated Structures, John Wiley \& Sons, 544 pp.

Pakrashi, V., Schoefs, F., Memet, J. B. \& O'Connor, A. 2008, "An Image Analysis Based Damage Classification Methodology", Structure and Infrastructure Engineering: Maintenance, Management, Life-Cycle Design and performance (NSIE), Taylor \& Francis (editors).

Rouhan, A. \& Schoefs, F. 2003, “Probabilistic modeling of inspection results for offshore structures", Journal of Structural Safety, Vol. 25, pp. 379-399.

Rudlin, J., Muhammed, A. \& Schneider, C. 2006, "Inspection reliability and periodicity for rail axle inspection", Insight, Vol. 48, No. 6, June, pp. 348-151.

Schoefs, F. \& Clement, A. 2004, “Multiple inspection modeling for decision making and management of 
jacket off-shore platforms: effect of false alarms", First International Forum on Engineering Decision Making (IFED'04), 5-9 December, Stoos, Switzerland.

Schoefs, F., Clément, A., Memet, J. B. \& Nouy, A. 2007a, "Spatial dependence of Receiver Operating Characteristic curves for Risk Based Inspection of corroded structures: application to on-pile wharf", Proceedings of $10^{\text {th }}$ international conference on Applications of Statistics and Probability in Civil Engineering, (I.C.A.S.P'07), Kanda, J., Takada, T. \& Furuta, H. (editors), Tokyo, Japan, 31 July to 3 August.

Schoefs, F., Yáñez-Godoy, H. \& Nouy, A. 2007b, "Identification of random material properties from monitoring of structures using stochastic chaos", Proceedings of $10^{\text {th }}$ International Conference on Applications of Statistics and Probability in Civil Engineering, (I.C.A.S.P'07), Kanda, J., Takada, T. \& Furuta, H. (editors), Tokyo, Japan, 31 July to 3 August.
Schoefs, F., Clément, A. \& Nouy, A. (submitted), "Assessment of spatially dependent ROC curves for inspection of random fields of defects", submitted to Structural Safety, 45 pp.

Sheils, E., O'Connor, A., Breysse, D., Schoefs, F. \& Yotte, S. 2008, "Development of a two stage inspection process for the assessment of deteriorating bridge structures", Journal of Bridge Engineering, publication of the American Society of Civil Engineers, Ed. Ref BE/2007/023379, to be published.

Silk, M. G. 1996, "Estimates of the probability of detection of flaws in TOFD data with varying levels of noise", Insight, Vol. 38, No. 1, January, pp. 31-36.

Straub, D. \& Faber, M. H. 2003, "Modeling dependency in inspection performance", Proc. Of the International Conference on Applications of Statistics and Probability in Civil Engineering (ICASP 03), Rotterdam. 